$2004 / 12 / 24$

\author{
Alexander E. Lobkovsky \\ Department of Earth, Atmospheric and Planetary Sciences, \\ Massachusetts Institute of Technology, Cambridge, MA 02139, USA \\ Bill Jensen and Arshad Kudrolli \\ Department of Physics, Clark University, Worcester, MA 01610, USA \\ Daniel H. Rothman \\ Department of Earth, Atmospheric and Planetary Sciences, \\ Massachusetts Institute of Technology, Cambridge, MA 02139, USA
}

\title{
Threshold phenomena in erosion driven by subsurface flow
}

\begin{abstract}
We study channelization and slope destabilization driven by subsurface (groundwater) flow in a laboratory experiment. The pressure of the water entering the sandpile from below as well as the slope of the sandpile are varied. We present quantitative understanding of the three modes of sediment mobilization in this experiment: surface erosion, fluidization, and slumping. The onset of erosion is controlled not only by shear stresses caused by surfical flows, but also hydrodynamic stresses deriving from subsurface flows. These additional forces require modification of the critical Shields criterion. Whereas surface flows alone can mobilize surface grains only when the water flux exceeds a threshold, subsurface flows cause this threshold to vanish at slopes steeper than a critical angle substantially smaller than the maximum angle of stability. Slopes above this critical angle are unstable to channelization by any amount of fluid reaching the surface.
\end{abstract}

\section{Introduction}

Unlike water, a layer of sand will not flow unless its surface is inclined beyond a characteristic angle, known as the maximum angle of stability [Duran, 1999]. This simple fact translates into a host of threshold phenomena wherever granular material is found. Many such phenomena play a crucial role in the erosion of Earth's surface, and very likely manifest themselves in the richness of the patterns exhibited by drainage networks.

Depending on geological, hydrological, and climatological properties, erosion by water is mainly driven either by overland flow or subsurface flow. The former case occurs when the shear stress imposed by a sheet flow exceeds a threshold [Horton, 1945; Loewenherz, 1991; Dietrich et al., 1992; Montgomery and Dietrich, 1992; Howard, 1994]. Erosion in the latter case - known as seepage erosion, or sappingoccurs when a subsurface flow emerges on the surface. Here the eroding stresses derive not only from the resulting sheet flow but also the process of seepage itself [Iverson and $\mathrm{Ma}$ jor, 1986]. The onset of erosion for both overland flow and seepage is threshold-dependent, but the additional source of stress in the case of seepage has the potential to create significantly different erosive dynamics.

Here we study the seepage case. Whereas the case of Horton overland flow has been extensively studied [Smith and Bretherton, 1972; Dietrich et al., 1992; Montgomery and Dietrich, 1994; Dunne et al., 1995; Izumi and Parker, 1995, 2000], seepage erosion has received less attention. Dunne [1980, 1990] suggests that erosive stresses due to seepage are more widespread in typical environments than commonly assumed. He also provides a detailed description of seepage erosion in the field, together with a discussion of the various factors that influence its occurrence. Another

Copyright 2018 by the American Geophysical Union. 0148-0227/18/2004JF000172 $\$ 9.00$ focus of attention has been the controversial possibility that many erosive features on Mars appear to have resulted from subsurface flows [Baker, 1982; Higgins, 1982; Laity and Malin, 1985; Baker, 1990; Aharonson et al., 2002]. Although the importance of seepage stresses in erosion have been realized by Howard [1988] and Howard and McLane [1988], comprehensive quantitative understanding is difficult to obtain. The complexity arises from the interdependent motion of the sediment and fluid - the "two-phase phenomenon" [Yalin, 1977] — which, of course, is common to all problems of erosion.

To further understand seepage erosion, we proceed from experiments [Schumm et al., 1987]. Questions concerning the origin of ancient Martian channels have motivated considerable experimental work in the past [Kochel et al., 1985; Kochel and Piper, 1986; Howard and McLane, 1988; Kochel et al., 1988]. The process of seepage erosion has also been studied as an example of drainage network development [Gomez and Mullen, 1992]. Our experiments, following those of Howard and McLane [1988]; Owoputi and Stolte [2001] and others, are designed to enable us to construct a predictive, quantitative theory. Consequently, they stress simplicity and completeness of information. Although our setup greatly simplifies much of Nature's complexity, we expect that at least some of our conclusions will improve general understanding, and therefore be relevant to real, fieldscale problems.

A previous paper by Schörghofer et al. [2004] provided a qualitative overview of the phenomenology in our experiment. It described the main modes of sediment mobilization: channelization, slumping, and fluidization. Here we provide quantitative understanding of the onset and transitions between these modes.

Our emphasis is on the threshold phenomena associated with the onset of erosion, which we will ultimately characterize in the same way that others [Duran, 1999] have characterized the onset of dry granular flow beyond the maximum angle of stability. This involves a construction of a generalized Shields criterion [Howard and McLane, 1988] valid 
in the presence of seepage through an inclined surface. A major conclusion is that the onset of erosion driven by seepage is significantly different from the onset of erosion driven by overland flow. We find that there is a critical slope $s_{\mathrm{c}}$, significantly smaller than the maximum angle of stability, above which the threshold disappears. Therefore any slope greater than $s_{\mathrm{c}}$ is unstable to erosion if there is seepage through it. This result is similar to well-known conclusions for the stability to frictional failure of slopes with uniform seepage [Taylor, 1965; Iverson and Major, 1986; Howard and McLane, 1988]. An important distinction in our work, however, concerns the mode of sediment mobilization and its local nature. The existence of the critical slope for seepage erosion may provide a useful quantitative complement to the qualitative distinctions between seepage and overland flow that have already been identified [Laity and Malin, 1985].

The remaining modes of sediment mobilization, fluidization and slumping, are modeled using well established ideas [Iverson and Major, 1986]. The result of applying these ideas together with the generalized Shields criterion provides a theoretical prediction of the outcomes of the experiment, i.e., a phase diagram. Agreement between theory and experiment is qualitative rather than quantitative. We nevertheless believe that our theoretical approach is fundamentally sound and that better agreement would follow from improved experimental procedures.

\section{Experiment}

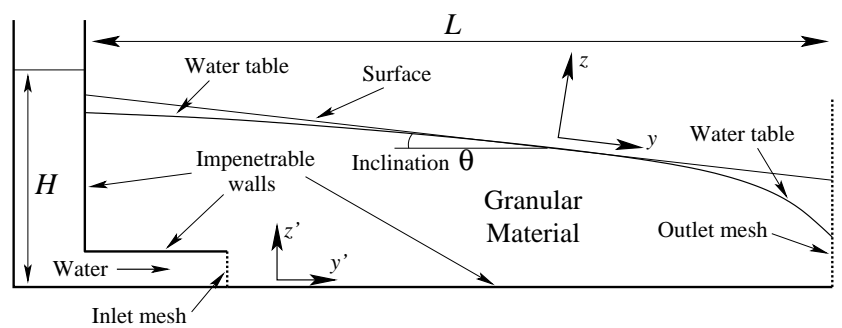

Figure 1. Schematic of the experiment as detailed in Schörghofer et al. [2004] as well as the setup for computing the bulk and surface water flow.

In our experimental setup, first introduced by Schörghofer et al. [2004], a pile of identical cohesionless glass beads $0.5 \mathrm{~mm}$ in diameter is saturated with water and compacted to create the densest possible packing. It is then shaped into a trapezoidal wedge inclined at an angle $\theta$ with slope $s=\tan \theta$ as shown in Fig. 1. The downslope length of the wedge is $L=90 \mathrm{~cm}$, its width across the slope is $119 \mathrm{~cm}$, and its height in the middle is approximately $11 \mathrm{~cm}$. Water enters the sandpile underneath through a fine metal mesh and exits at the lower end of the pile through the same kind of mesh. A constant head at the inlet is maintained by keeping a constant water level $H$ in the reservoir behind the sandbox with the help of an outflow pipe. The slope $s$ of the pile and the water level $H$ are the control parameters of the experiment. The degree of packing of the granular pile is the variable most difficult to control.

Our particular method of feeding water into the sandpile, similar to that of Owoputi and Stolte [2001], can be motivated in three ways. The most important justification is the fact that the amount of water flowing on the surface can be finely controlled in our geometry. This feature is essential in probing the onset of erosion. Second, our setup allows us to access heads $H$ larger than the height of the pile, which therefore allows us to explore dynamic regimes unavailable if water enters the pile through a mesh in the back. Third, a similar seepage water flow geometry can exist in the field wherever water travels beneath an impermeable layer that terminates.

We have performed two types of experiments: steady and non-steady. For a fixed water level and in absence of sediment motion, water flow reaches steady state. By monitoring the total water flux through the system we estimate the time to reach steady state to be approximately ten minutes. To explore the onset of sediment motion, we raised the water level $H$ in small increments, waiting each time for steady state to be established. Due to the particular shape of the bulk flow in our experiment, surface flow exists over a finite region of the surface. The width of this seepage face and therefore the depth of the surface flow can be tuned by changing $H$. Because of the finite extent of surface flow, its depth and therefore the viscous shear stress reaches a maximum at a certain location. Thus, by increasing $H$ we can continuously tune the maximum shear stress experienced by the surface grains. The maximum shear stress reaches a critical value for the onset of sediment motion in a certain location on the slope. As we show below, we can compute where the maximum shear stress occurs and thus can reliably detect the onset of sediment motion visually because we focus our attention on this location. Once sediment begins to move, channels form almost immediately. These channels grow in length, width, and depth. An example of the evolving channel network is shown in Fig. 2. Depending on the slope, as the channels deepen, the pile becomes unstable to fluidization or slumping. For slopes lower than approximately 0.05 , the fluidization threshold is reached before sediment is mobilized on the surface.

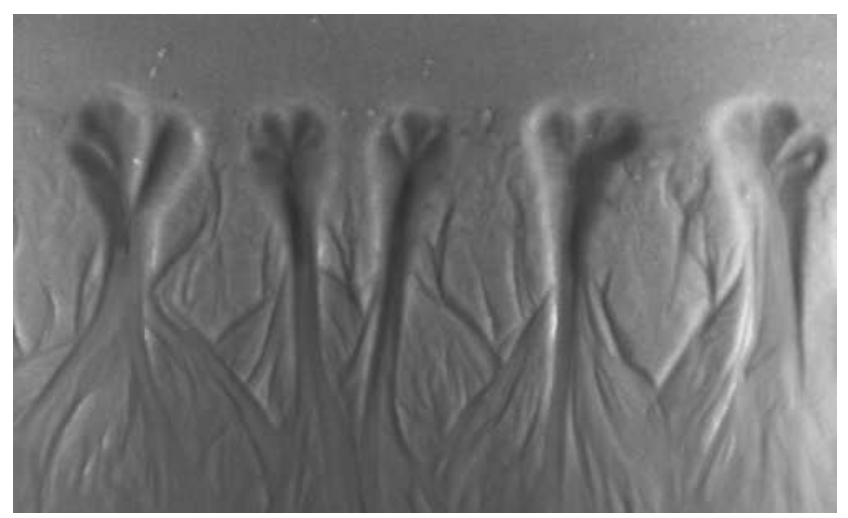

Figure 2. Examples of the channel network. Top view of the slope of the eroding sandpile. Branched channel heads migrate up the slope (upward) while the eroded sediment is deposited in the braided wash downslope. The horizontal size of the image is approximately $1 \mathrm{~m}$. The slope of the pile is $s=0.1$ and the water level $H=16.15 \mathrm{~cm}$.

We also explored the non-steady evolution of the bulk and surface water flow and resulting sediment motion by raising the water level $H$ to some higher value from zero. In this case one of three things can happen. The pile can be fluidized within a few seconds or fail by slumping as shown in Fig. 3. If this does not occur, the water emerges on the surface just above the inlet. A sheet of water then washes down the slope of the pile. During this initial wash, sediment is mobilized and incipient channels form. These channels grow during subsequent relaxation of the bulk water flow towards steady state. Because of the initial wash's erosive power, 
channels are able to form and grow for lower water pres-

sures than in steady experiments.

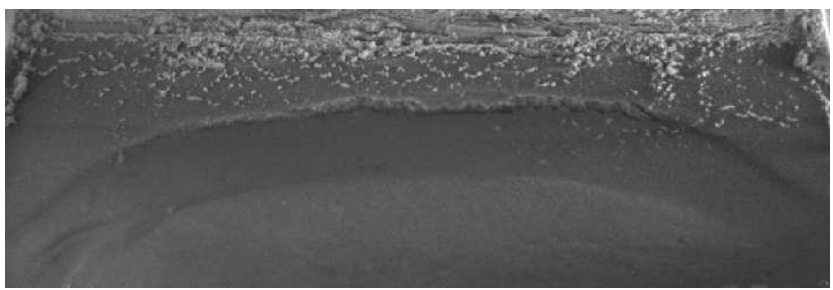

Figure 3. Example of a slumping sandpile viewed at an approximately $45^{\circ}$ angle to the slope after the water flow has been stopped. The width of the imaged region is approximately $1 \mathrm{~m}$. Slumping happens along a convex upward arc which looks darker because it is deeper and therefore wetter.

Outcomes of a large number of non-steady experiments

and several steady experiments for varying slope $s$ and the

water level $H$ are summarized in the phase diagram in Fig.

4. Each symbol in the plot represents one experiment. The

sediment is either immobile (stable seepage), or it is mobi-

lized on the surface where channels form (channelization)

or in the bulk (slumping or fluidization). In several ex-

periments, slumping or fluidization happened after chan-

nels formed and grew. In the following sections we describe

the computations that allow us to construct the theoretical

boundaries between the three different modes of sediment

mobilization in our experiment.

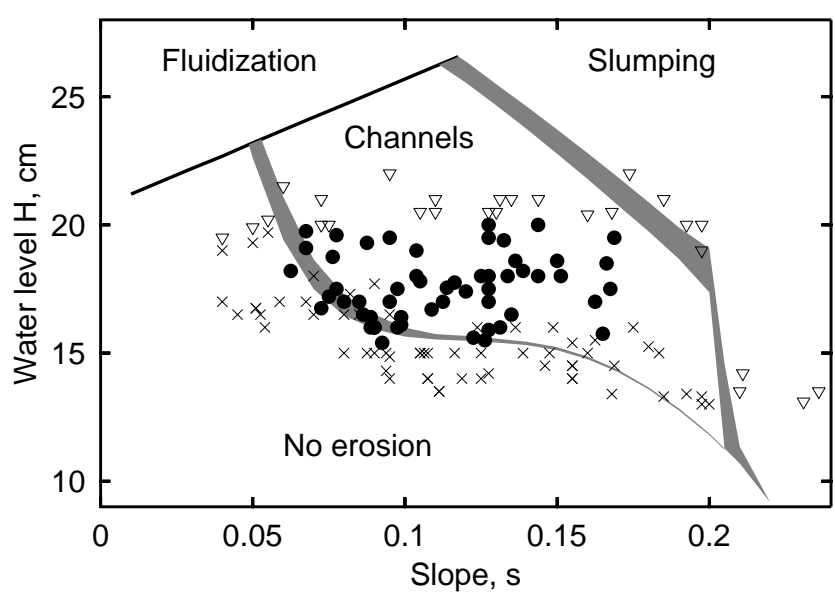

Figure 4. Experimental and theoretical phase diagram in the parameter space of slope and water level. Experiments that yielded no erosion are denoted by $\times$; those that produced channels are indicated by $\bullet$; and those that produced fluidization and/or slumping within one hour of the beginning of the experiment are represented by $\nabla$. The straight line and gray-shaded curves are theoretical predictions for the boundaries separating the four regions indicated by their labels. The thickness of the lines indicates uncertainty in the theory. The boundary between the uneroded and channelized states is reasonably well approximated by our theory. The theoretical boundaries for fluidization and slumping, however, appear to overestimate the critical water level, possibly as a result of inhomogeneities, dynamic changes in the sandpile's shape, or from the assumption of a steady state.

\section{Calculation of the water flow}

Whereas steady-state flow can be readily characterized quantitatively, non-steady flow characterization requires knowledge of the water-table dynamics. However, the theory of the water-table dynamics is less well established than that of the flow through the bulk of a porous medium. Also, our steady-state experiments probe all aspects of sediment dynamics. We can therefore focus on the quantitative characterization of the steady-state flow.

To study the onset of erosion quantitatively we need to be able to establish a correspondence between the experimentally measurable quantities such as the slope $s$, the water level $H$, the size of the seepage face, and the water fluxes. The seepage and surface fluxes are the most difficult to measure. In this section we set up their computation. The computation is designed to enable us to infer water fluxes indirectly by measuring the size of the seepage face. In the following sections we will use this computation to quantify the onset of erosion and to compute the slumping and liquefaction boundaries of the channelization phase diagram shown in Fig. 4.

Fig. 1 specifies the key quantities and coordinate systems we use in computing the fluxes. The flow profile is independent of the $x$-coordinate across the slope of the sandpile except near the side walls of the box. We therefore treat the box as if it were infinitely wide. Flow is then twodimensional and the specific discharge vector $\mathbf{q}$ is in the $y-z$ plane. We will use two coordinate systems. As shown in Fig. 1 , the $z^{\prime}$ coordinate is measured vertically from the bottom of the box while the $z$ coordinate is the normal distance away from the surface of the pile. The flow is governed by Darcy's law,

$$
\mathbf{q}=-K \nabla \psi
$$


where $K$ is the scalar hydraulic conductivity, and

$$
\psi=\pi+z^{\prime}
$$

is the total hydraulic head of the pore water. Both $\mathbf{q}$ and $K$ have units of velocity while the scaled pore pressure $\pi=p /(\rho g)$ has units of length. Here $\rho$ is the density of water and $g$ is the magnitude of the acceleration of gravity. We have measured $K$ via a U-tube relaxation experiment. To do so we created a water level difference $\Delta h$ between the two arms of a transparent $U$-shaped tube of width $\ell$ partially filled with glass beads. The rate of change of $\Delta h$ is given by $K \Delta h / \ell$. By measuring the rate of change of $\Delta h$ we deduced the value of the hydraulic conductivity $K=3.0 \pm 0.1 \mathrm{~mm} / \mathrm{s}$ (Schörghofer et al. [2004]). Hydraulic conductivity is sensitive to the packing of the grains and is the variable most difficult to control in our experiment.

Water incompressibility implies $\nabla \cdot \mathbf{q}=0$, therefore yielding Laplace's equation,

$$
\nabla^{2} \pi=0
$$

To compute the pore pressure $\pi$, boundary conditions must be specified. The walls of the box are impenetrable. Therefore the discharge vector is parallel to the walls. In other words, the flux $q_{\perp}$ in the direction $\hat{\mathbf{n}}$ normal to the walls vanishes. Thus $q_{\perp} \equiv \hat{\mathbf{n}} \cdot \mathbf{q}=0$. Because the glass beads in our experiment are small, capillarity is important. When a tube filled with glass beads is lowered into a reservoir of water, the porous bead-pack fully saturates in a region that extends above the surface of the water by a capillary rise $\pi_{\mathrm{c}}$. We measured $\pi_{\mathrm{c}}=25 \mathrm{~mm}$ for our material. The capillary rise is a measure of the average radius of the water menisci at the edge of the fully saturated zone. The pore pressure at the edge of the fully saturated zone is $-\pi_{\mathrm{c}}$ (without loss of generality we set the atmospheric pressure to zero). Water can rise above the fully saturated zone through the smaller pores and narrower throats. Thus a partially saturated capillary fringe exists above the fully saturated zone. However, in this fringe the water is effectively immobile since it is confined to the smaller pores and narrower throats.

Since water flows only in the fully saturated zone, we define the water table to be at its edge. Thus, the pore pressure at the water table is equal to the negative capillary rise $\pi=-\pi_{\mathrm{c}}$. In steady state the discharge vector is parallel to the water table. This extra condition allows us to determine the location of the water table in steady state.

We neglect the pressure drop across the inlet mesh. Therefore, the pore pressure at the inlet mesh is $\pi=H-z^{\prime}$. The boundary conditions at the surface of the sandpile and at the outlet mesh are more subtle. When no water seeps out, i.e., when the discharge vector is parallel to the surface, the curvature of the water menisci between grains can freely adjust so that the pressure $\pi$ can vary between zero and $-\pi_{\mathrm{c}}$. Therefore when $-\pi_{\mathrm{c}}<\pi<0$, no seepage occurs. Otherwise, the pore pressure equals the atmospheric pressure $\pi=0$ (we neglect the pressure exerted by the thin layer of water on the surface), and the discharge vector has a com- ponent normal to the surface, i.e., there is either exfiltration or infiltration.

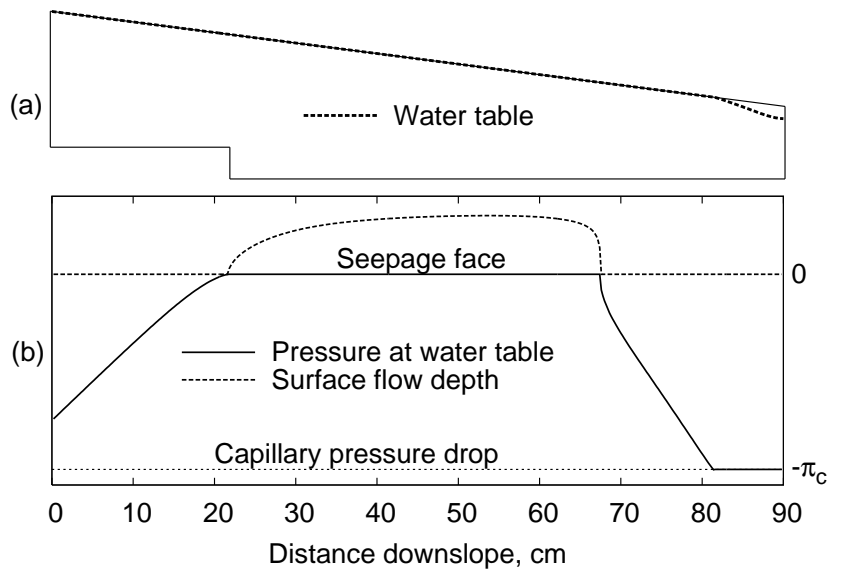

Figure 5. (a) Steady state location of the water table (dashed line) obtained by solving the Laplace's equation as detailed in the text. Note that the water table is almost entirely at the surface of the sandpile except a for small region at the bottom of the sandpile. (b) Pore pressure at the water table and surface flow. Vertical scale for the pressure at the water table is given by the negative capillary rise $-\pi_{\mathrm{c}}$, or the pressure at the water table when it is below the surface of the pile. Note that seepage occurs only where the pore pressure reaches atmospheric pressure. Slope is $s=0.1$, water level $H=15 \mathrm{~cm}$.

To obtain the steady state location of the water table, we guess its position and solve Laplace's equation (3) with the $\pi=-\pi_{\mathrm{c}}$ boundary condition on the water table. We then move the water table in the direction of the local discharge vector by an amount proportional to its length. Iteration of this procedure converges to the steady-state position of the water table. An example is shown in Figure 5.

Once the steady flow pattern is known, we can calculate the overland water flux $Q(y)$ by integrating the one-dimensional continuity condition which states that the downslope derivative of the overland flux is equal to the seepage flux:

$$
\frac{d Q}{d y}=q_{z} \equiv \hat{\mathbf{z}} \cdot \mathbf{q}=-\left.K \frac{\partial \psi}{\partial z}\right|_{y=0}
$$

\section{Onset of erosion and channel incision}

In this section we assume, based on direct observation, that the onset of channel incision coincides with the onset of erosion (i.e., we never observed a homogeneously eroding state). In other words, when the overland water flux becomes strong enough to carry grains, the flow of sediment becomes immediately unstable to perturbations transverse to the downslope direction and incipient channels form [Smith and Bretherton, 1972]. Using this assumption and the calculation of the overland water flux we can deduce the threshold condition for the onset of erosion.

It is universally assumed after Shields [1936] that the hydrodynamic stresses exerted on the sandpile by the fluid flowing on its surface determine whether cohesionless granular material is entrained. In the limit of laminar flow, the dominant hydrodynamic stress is the viscous shear stress $\tau_{\mathrm{b}}$. 
Appropriately scaled this shear stress is termed the Shields number [Yalin, 1977], defined by

$$
\tau^{*}=\frac{\tau_{\mathrm{b}}}{\left(\rho_{\mathrm{s}}-\rho\right) g d}
$$

where $\rho_{\mathrm{s}}$ is the density of the granular material, $d$ is the grain diameter $(d=0.5 \mathrm{~mm}$ in our experiment), and the surface is not inclined.

The conventional Shields number (5) is the ratio between the horizontal force exerted by the flow and vertical force due to grain's weight. To generalize the notion of the Shields number to the situation with seepage through an inclined surface, we make two changes in Eq. (5). We first add the tangential component of the seepage force density $\mathbf{f}=-\rho g \nabla \psi$ acting over a length $d$ to the numerator of (5). The numerator thus becomes $\tau_{\mathrm{b}}+d(\hat{\mathbf{y}} \cdot \mathbf{f})$. Note that we did not include the tangential component of the grain's weight to the numerator. Defined in this way, the generalized Shields number measures the effect of the fluid: both the bulk as well as the surface flows.

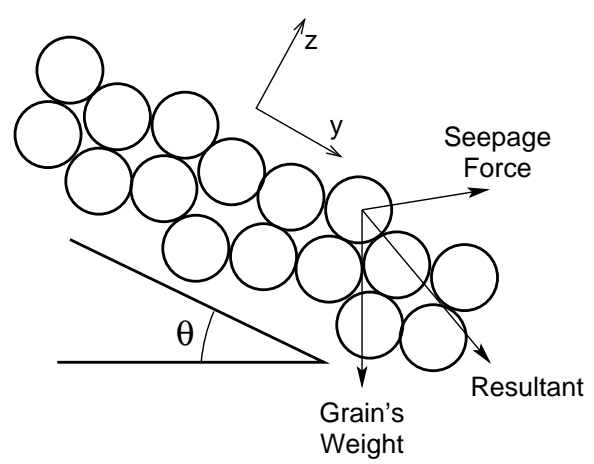

Figure 6. The denominator of the generalized Shields number is the projection onto the $z$-axis of the resultant of the grain's weight and the seepage force both scaled by $\pi d^{2} / 6$.

Second, we replace the denominator of Eq. (5) by the resultant (vectorial sum) of the seepage force on a grain and its submerged weight, as shown in Fig. 6, both scaled by $\pi d^{2} / 6$ ( $d^{2}$ to obtain stress as in the numerator and $\pi / 6$ for agreement with the conventional Shields number), projected onto the $z$-axis. According to Martin and Aral [1971] the grains on the surface of the bed experience a seepage force roughly half as large as the grains several layers deep. Consequently, we assume that the seepage force is reduced by a factor of $a \approx 0.5$; therefore

$$
\tau^{*}=\frac{\tau_{\mathrm{b}}-a \rho g d(\partial \psi / \partial y)}{\left(\rho_{\mathrm{s}}-\rho\right) g d \cos \theta+a \rho g d(\partial \psi / \partial z)},
$$

where $\theta$ is the inclination angle of the surface. The importance of the seepage stresses for the criterion for the onset of erosion was previously realized by Howard and McLane [1988]. It can be shown that their equation (10) expressing marginal stability of a surface grain is equivalent to writing $\tau^{*}=\tan \phi$, the tangent of the angle of internal friction.

The generalized Shields number Eq. (6) is a measure of the relative importance of the tangential and normal forces acting on a grain at the surface of the sandpile. Therefore, we expect $\tau^{*}$ to be a control parameter for erosion. In other words, there exists a critical Shields number $\tau_{\mathrm{c}}^{*}$, such that when $\tau^{*}<\tau_{\mathrm{c}}^{*}$, surface grains are immobile, and when $\tau^{*}>\tau_{\mathrm{c}}^{*}$, sediment is mobilized. Note that (6) reduces to the classical definition of the Shields number for a flat surface without seepage. Also note that since we did not include the tangential component of grain's weight, the critical Shields number at the onset of sediment motion vanishes when the inclination angle $\theta$ reaches the maximum angle of stability.

Although we obtain the seepage force density $\mathbf{f}$ as a result of computing the pore-water pressure, to calculate the boundary shear stress $\tau_{\mathrm{b}}$ we must estimate the thickness of the surface water layer $h(y)$. Since this thickness changes slowly in the downslope direction, we can approximate the surface flow by the steady flow of a uniform layer of viscous fluid. Also, the surface water flux is small enough for turbulence to be of no importance. The thickness $h$ of laminar surface flow for a given flux $Q$ is [Landau and Lifshitz, 1987]

$$
h=\left(\frac{3 Q \eta}{\rho g \sin \theta}\right)^{1 / 3},
$$

where $\eta$ is the viscosity, while the viscous shear stress exerted on the sandpile is

$$
\tau_{\mathrm{b}}=\rho g h \sin \theta
$$

The particle Reynolds number can then be calculated using the bottom shear stress $(8)$ and shear velocity $v^{*}=\sqrt{\tau_{\mathrm{b}} / \rho}$ as

$$
\operatorname{Re}=\frac{v^{*} d}{\nu}
$$

where $\nu=\eta / \rho$ is the kinematic viscosity of water. We estimate that in our experiments, this particle Reynolds number varies between 5 and 20 depending on the slope $s$ of the pile and the water level $H$. We verify this estimate of the Reynolds number by a direct measurement of the thickness of the surface flow. We find that this thickness is several grain diameters. This justifies the laminar flow assumption used in obtaining Eq. (7).

Using (8), the Shields number (6) can now be conveniently rewritten as

$$
\tau^{*}=\frac{(h / d) \sin \theta-a(\partial \psi / \partial y)}{\left(\rho_{\mathrm{s}} / \rho-1\right) \cos \theta+a(\partial \psi / \partial z)} .
$$

This expression can be further simplified by noting that $\pi=0$ along the seepage face. Therefore $\partial \psi / \partial y=-\sin \theta$ at the surface wherever there is overland flow. We arrive at the final expression for the modified Shields number which depends on the surface flow thickness $h$, the normal component $\partial \psi / \partial z$ of the seepage force density at the surface, and the seepage force reduction factor $a$

$$
\tau^{*}=\frac{(h / d+a) \sin \theta}{\left(\rho_{\mathrm{s}} / \rho-1\right) \cos \theta+a(\partial \psi / \partial z)} .
$$

In our geometry, both the surface and the seepage water fluxes reach a maximum somewhere along the slope. Therefore the Shields number has a maximum value as well. Below we calculate this maximum Shields number in steady state for a given slope $s$ and water level $H$.

\section{Critical slope for the onset of seepage erosion}




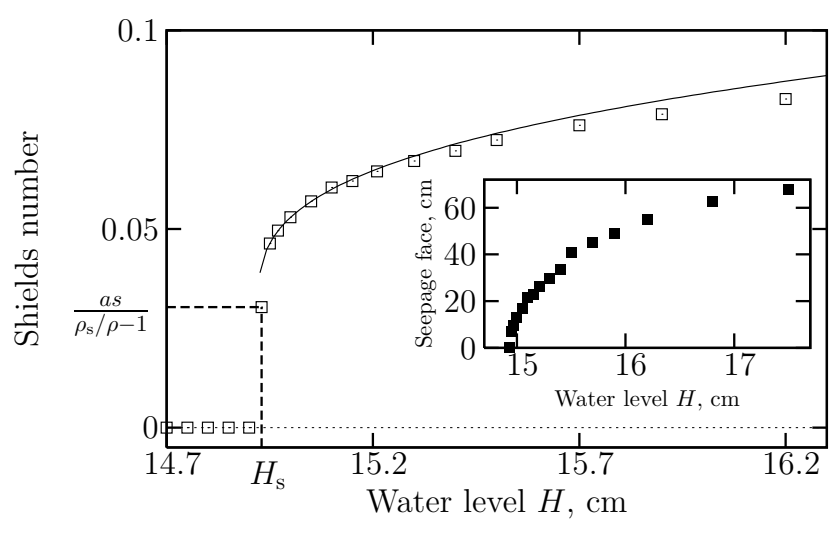

Figure 7. Computed maximum Shields number versus the water level $H$ for a pile of slope $s=0.1$. At $H=H_{\mathrm{s}}$ water first seeps through the surface and the Shields number jumps to a nonzero value. Afterwards it increases rapidly as $\left(H-H_{\mathrm{s}}\right)^{1 / 3}$ (solid line). Inset: corresponding size of the computed seepage face.

We now explore the consequences of seepage for the phenomenology of the onset of erosion. Because of the additional force on the surface grains, seepage flow is more erosive than overland flow. This notion is reflected quantitatively in the generalized Shields number. Let us examine how the maximum Shields number varies with the water level $H$ in our experiment. A representative graph of the maximum Shields number versus the water level is shown in Fig. 7. Below a water level $H_{\mathrm{s}}(s)$ that is a function of the slope, no water seeps out to the surface of the pile. Even though the water table may be at the surface, the pressure at the water table is below atmospheric pressure and capillarity prevents seepage. When $H=H_{\mathrm{s}}$, i.e., exactly at the onset of seepage, the pressure reaches $\pi=0$ at some point on the surface. Since the seepage flux is still zero, $\partial \psi / \partial z=0$ along the wet part of the surface. Therefore, just above the seepage onset, when the water layer thickness and the seepage flux are both infinitesimally small, the maximum Shields number is

$$
\tau_{\mathrm{s}}^{*}(s)=\frac{a s}{\rho_{\mathrm{s}} / \rho-1} .
$$

In contrast to overland flow, the consequence of seepage is that as soon as the water emerges on the surface, the maximum Shields number is some non-zero value which depends on the slope. This also implies that there exists a critical slope $s_{\mathrm{c}}$ such that $\tau_{\mathrm{s}}\left(s_{\mathrm{c}}\right)$ is equal to the critical Shields number $\tau_{\mathrm{c}}^{*}$, i.e.,

$$
s_{\mathrm{c}}=\tau_{\mathrm{c}}^{*}\left(\rho_{\mathrm{s}} / \rho-1\right) / a
$$

For slopes greater than $s_{\mathrm{c}}$ seepage is always erosive. Note that for low-density particles this critical slope can be arbitrarily small. The expression for the critical slope for seepage erosion in Eq. (13) is analogous to well-known formulas for stability of slopes to Coulomb failure due to uniform seepage [Iverson and Major, 1986]. Our result applies locally to the point where non-uniform seepage first emerges on the surface. In this situation, the pile is generally stable to Coulomb failure and the sediment is eroded only locally on the surface.

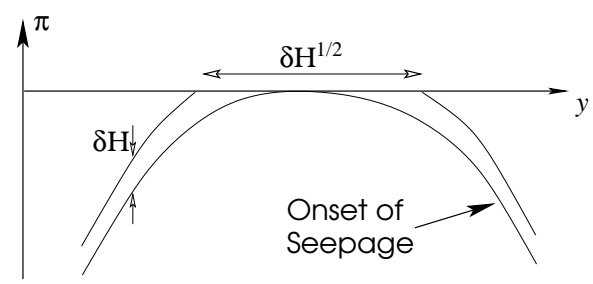

Figure 8. Schematic of the pore pressure $\pi$ as a function of the downslope coordinate $y$ at the onset of seepage $H=H_{\mathrm{s}}$ and just above.

We now show that above $H_{\mathrm{s}}$, the maximum Shields number increases rapidly as a $1 / 3$ power of the water level excess $\left(H-H_{\mathrm{s}}\right)^{1 / 3}$. At the onset of seepage, i.e., when $H=H_{\mathrm{s}}$, the pressure at the water table reaches atmospheric pressure $\pi=0$ at some point $P$ located at $z=0$ and $y=y_{\mathrm{s}}$ on the surface. Even though the water table is at the surface, there is no seepage anywhere, i.e., $\partial \psi / \partial z=0$. Because the pressure is smooth, it can be approximated by a quadratic function near this point so that $\pi \approx b z^{2}-c\left(y-y_{\mathrm{s}}\right)^{2}$, where $b$ and $c$ are constants with appropriate dimensions. When the water level is raised by a small increment $\delta H=H-H_{\mathrm{s}}$, the lowest order change in the head at the water table is an increase of $\delta H$ with the exception of the region where this increase would lead to a positive pressure. As illustrated in Fig. 8 , in this region the pore pressure $\pi$ is set to 0 , and thus this region becomes the seepage face. The width of the seepage face $W$ scales like the square root of $\delta H$, i.e., $W \sim \delta H^{1 / 2}$ as seen in the inset of Fig. 7. The seepage flux can be estimated by noting that the hydraulic head is modified by an amount $\delta H$ over a vertical region of order $W$. Therefore we obtain $\partial \psi / \partial z \sim \delta H / W \sim \delta H^{1 / 2}$. The total surface flux therefore scales like the product of the seepage flux and the width $W$ of the seepage face, i.e., $Q \sim W \partial \psi / \partial z \sim \delta H$. The lowest order change in the maximum Shields number is due to the change of the surface flow depth $h \sim Q^{1 / 3} \sim \delta H^{1 / 3}$. Thus as we claimed above, just above the water level $H_{\mathrm{s}}$ for the onset of seepage,

$$
\tau^{*} \approx \tau_{\mathrm{s}}^{*}(s)+\alpha(s)\left(H-H_{\mathrm{s}}\right)^{1 / 3},
$$

where the constant $\alpha$ is a function of the slope. Variation with water level of the computed maximum Shields number shown in Fig. 7 is consistent with Eq. (14).

\section{Measurement of the critical Shields number}

In the previous sections we have detailed the way of calculating the bulk and surface water fluxes in our experiment and the resulting maximum generalized Shields number. In this section we use this calculation to examine the onset of the sediment flow and channelization. Our first goal is to measure the threshold or critical Shields number required for the mobilization of sediment. We then use this measured value of the critical Shields number to predict the outcome of steady-state experiments for various values of the slope and the water level and thus compute the channelization boundary in the phase diagram in Fig. 4.

The actual maximum Shields number in the experiment differs from the quantity calculated in Eq. (11). In addition to random errors in the measurements of the pile dimensions and water level, there are several sources of systematic error. 
For example, the pressure drop across the inlet mesh results in a lower effective hydraulic head. Also, our measurement of the capillary rise $\pi_{\mathrm{c}}$ is dependent on a visual estimate of the fully saturated zone and thus can be a source of systematic error. We indeed find that the size of the seepage face calculated for a particular water level $H$ is greater than measured in the experiment. However, the size of the seepage face translates directly into the surface water flux and therefore the maximum Shields number.

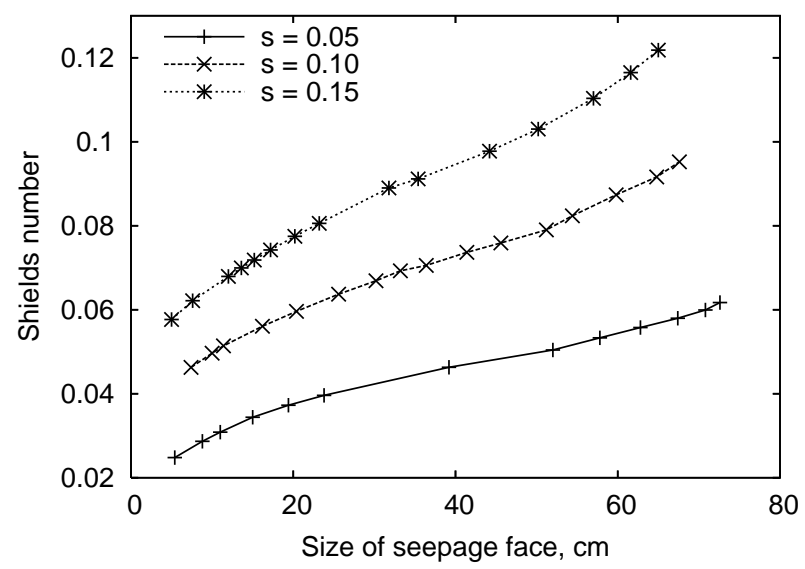

Figure 9. Computed maximum Shields number versus the seepage face size for three different slopes. We use this calculation to infer the Shields number from the experimentally measured size of the seepage face.

The inset of Figure 7 shows the typical dependence of the size of the seepage face on the water level. The variation of the maximum Shields number with the size of the seepage face is shown in Fig. 9 for three different slopes. We use this computed correspondence between the size of the seepage face and the maximum Shields number to infer the maximum Shields number in the experiment by measuring the size of the seepage face.

To measure the critical Shields number we raise the water level $H$ in increments of a few millimeters at a time. Each time the water level is increased, the seepage flow is allowed to reach a steady state. In each of these steady states we measure the seepage face size and infer the corresponding maximum Shields number. Eventually, sediment is mobilized and we record the size of the seepage face and compute the corresponding maximum Shields number. This number is an upper bound on the critical Shields number for our granular material at that particular slope. The lower bound on the critical Shields number is obtained from the largest seepage face at which no sediment is moving or sediment motion is only transient. Averaging over several experiments with slope $s=0.1$ we estimate the critical Shields number to be

$$
\tau_{\mathrm{c}}^{*}=0.085 \pm 0.005 \text {. }
$$

It is not obvious that the generalized critical Shields number for the onset of seepage driven erosion should coincide with the critical Shields number for overland flow. However our measured value of the critical generalized Shields number is within the scatter of the existing data for overland flow summarized in Buffington and Montgomery [1997]. Our measurement the critical generalized Shields number is equivalent to measuring the angle of internal friction due to the correspondence of our definition of $\tau^{*}(6)$ and Howard and McLane's equation (10).

Deviations from flatness of the pile's surface result in the fluctuations of the thickness of the surface water film. As a result, the maximum bottom shear stress in the experiment is systematically greater than that calculated at a given size of the seepage face. Thus the Shields number calculated for a particular size of the seepage face is the lower bound on the actual Shields number in the experiment.

In principle, the critical Shields number should vary with the slope of the pile. Evidence for this is the fact that at the maximum angle of stability any additional forcing from the water flowing over the bed mobilizes sediment. Since it is reasonable to assume that the critical Shields number is continuous and monotonic, we arrive at the notion that it decreases monotonically with slope and vanishes at the maximum angle of stability. For small slopes the critical Shields number is expected to decrease as the cosine of the inclination angle since this is the lowest order change in the stabilizing effect of gravity. For most slopes in our experiments, $\cos \theta$ is within a few percent of unity and thus we can ignore the variation of the critical Shields number with slope.

This assumption allows us to predict the water level at which erosion and therefore channelization should commence in our experiment. In Fig. 4 a boundary is drawn between regions where sediment is expected to mobilize and remain immobile. To obtain this line we computed for each slope the water level $H_{\mathrm{e}}(s)$ at which the Shields number is equal to the critical Shields number (15). Below this water level, i.e., when $H<H_{\mathrm{e}}(s)$, the maximum Shields number is below critical and thus sediment is immobile. Conversely, for $H>H_{\mathrm{e}}(s)$, the maximum Shields number is above critical and thus sediment is mobilized and channels form. The channelization boundary is widened because of the uncertainty in the critical Shields number.

Qualitative agreement of the channelization boundary with experiments is perhaps due to the opposite action of two effects. First, channelization occurs for lower water levels in non-steady experiments. This happens because in non-steady experiments the maximum Shields number overshoots its steady-state value. The overshoot is greatest for small slopes. Second, a pressure drop across the inlet mesh and the compacted region of sand close to it has an opposite effect which increases the water level needed for channelization. These two effects, though small, could together affect the accuracy of our predictions. Since these effects act in opposite ways, our the predictions of the calculated channelization water level $H_{\mathrm{e}}(s)$ agree qualitatively with the experiments.

\section{Fluidization and slumping}

Having computed the channelization boundary in the phase diagram, we now pursue a quantitative description of the other two modes of sediment mobilization exhibited by our sandpile. Higher water pressures can cause the sandpile to fail in one of two ways. First, an upward seepage force can lift sand and result in a fluidization or quicksand instability. Second, the pile can become unstable to slipping, slumping, or sliding. Both failure mechanisms have been discussed by a number of studies, e.g., those of Iverson and Major [1986] or Martin and Aral [1971]. 


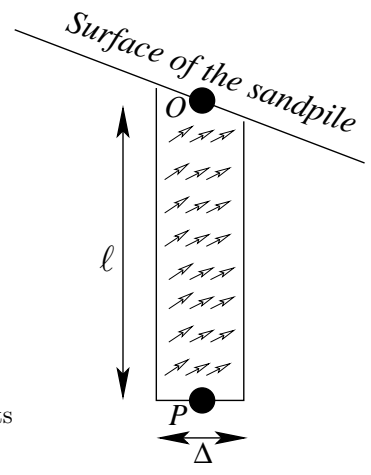

Figure 10. To predict fluidization, we calculate the total seepage force on a slice of width $\Delta$ and height $\ell$ and compare it with its weight.

Fluidization occurs when at some point $P$ in the sandpile the pore pressure is larger than the total hydrostatic pressure due to the weight of the sand and the water above $P$. To see this we compute the total seepage force acting on a slice of sand of width $\Delta$ between point $P$ and point $O$ on the surface of the pile directly above $P$. The vertical component of this force is (see Fig. 10)

$$
F_{\mathrm{v}}=\rho g \Delta\left(\psi_{\mathrm{P}}-\psi_{\mathrm{O}}\right)=\rho g \Delta\left(\pi_{\mathrm{P}}-\pi_{\mathrm{O}}-\ell\right),
$$

where $\ell$ is the height of the slice. When this force exceeds the submerged weight $\Delta \ell\left(\rho_{\mathrm{t}}-\rho\right) g$ of the slice, the slice is lifted and the bed is fluidized. Here $\rho_{\mathrm{t}}$ is the total density of the saturated sand, which for our sand is approximately $2 \mathrm{~g} / \mathrm{cm}^{3}$. Thus fluidization occurs when there exists points $P$ and $O$ on the surface directly above $P$ such that

$$
\pi_{\mathrm{P}}-\pi_{\mathrm{O}}>\ell \rho_{\mathrm{t}} / \rho
$$

For uniform seepage this condition is equivalent to those in Iverson and Major [1986] and Martin and Aral [1971].

To construct the fluidization boundary in the phase diagram (Fig. 4), we find the water level $H_{\text {fluid }}$ above which there exists at least one point in the pile for which condition (17) is satisfied. Below this fluidization water level this condition is not satisfied for any point in the pile.

In addition to fluidization the sandpile can fail by slumping. This can happen in one of two ways. Frictional failure can occur in the bulk of the pile due to the seepage stresses. Alternatively, surface avalanching can occur. To establish an upper bound on the water level at which the sandpile slumps via either mechanism we use the criterion developed by Iverson and Major [1986] for determining when a slope is destabilized by uniform groundwater seepage. Essentially it requires calculating the vectorial sum of the seepage and gravity forces acting on a small element of soil near the surface. When the angle between this total force and the downward normal to the surface, which we will call the effective inclination angle, exceeds the maximum angle of stability, the surface grains are destabilized. We measured the maximum angle of stability to be $\varphi \approx 23^{\circ}$ for dry glass beads. The slumping boundary in the phase diagram (Fig. 4 ) is constructed by computing the effective inclination angle along the surface of the pile and noting the water level $H_{\text {slump }}$, at which the effective inclination angle reaches the maximum angle of stability $\varphi$ at some point of the surface.

Figure 4 shows the critical water level at which fluidization and slumping should occur according to the criteria above. Failure occurs at systematically lower water levels in the experiment. There are several effects which can account for this difference. First, any irregularities in the construction of the pile such as voids or surface height fluctuations make the pile more unstable to fluidization and slumping.
Second, we compute the instability of an uneroded pile, whereas in most experiments, the pile failed after erosion had changed the shape of the pile. The decrease of pile's height due to erosion increases the head gradient in the bulk and thus makes the pile more prone to slumping and/or fluidization.

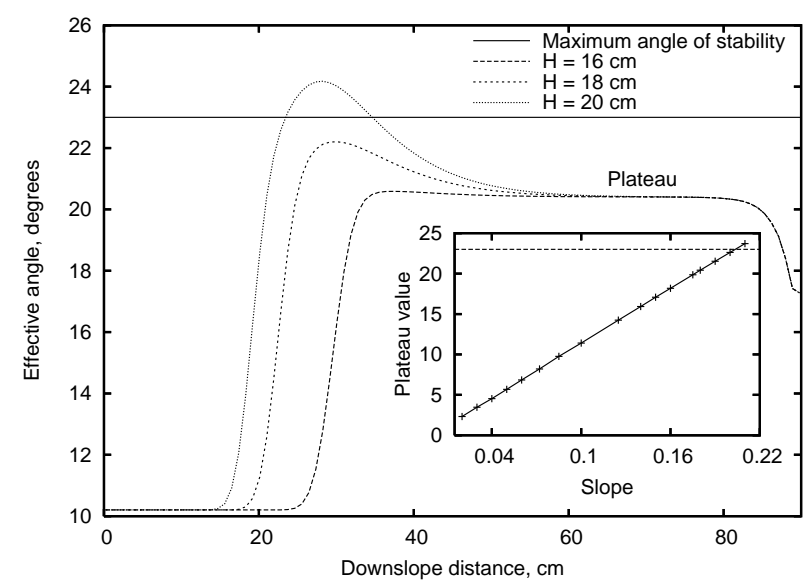

Figure 11. Effective inclination angle at the surface due to seepage forces for $s=0.18$ and three different water levels. For the highest water level, a region on the surface has an effective angle above the maximum angle of stability and thus the slope is unstable to slumping. The inset shows the plateau value of the effective inclination angle as a function of slope. When the plateau value reaches the maximum angle of stability, even a small amount of seepage destabilizes the pile to slumping.

At $s \approx 0.2$, a jump in the slumping water level is observed in both the experiment and the model. This jump is a purely geometric effect. Slumping occurs when, somewhere along the slope, the effective inclination angle, which includes the effect of the seepage force, exceeds the maximum angle of stability. As shown in Fig. 11, for slopes smaller than 0.2, the effective inclination angle is flat and develops a peak under the water inlet as the water pressure is increased. When the top of this peak crosses the value of the maximum angle of stability, the pile slumps. When the slope exceeds 0.2 , however, the value of the plateau in the effective inclination angle is above the maximum angle of stability. Therefore, for these slopes, the pile will be unstable to slumping as soon as the water emerges on the surface.

\section{Conclusions}

This article reports on our progress in understanding seepage erosion of a simple non-cohesive granular material in a laboratory-scale experiment introduced in Schörghofer et al. [2004]. Our ultimate goal is to construct a quantitative predictive theory of the onset and growth of the channel network observed in this experiment. This goal requires a complete sediment transport model as well as the calculation of the relevant water fluxes. Here we obtain the latter and focus on the onset of erosion.

Prediction of the onset of erosion based on the generalized Shields conjecture explains qualitatively the channelization boundary in the experimental phase diagram. By invoking well established simple ideas we also roughly explain the fluidization and slumping boundaries in the 
phase diagram. Greater discrepancy with the experiment for these boundaries indicates that better understanding of the slumping/fluidization mechanisms particular to our experiment is needed.

The central result of our exploration is the introduction of the generalized Shields criterion for seepage erosion. As a consequence of seepage forces on the surface grains, the threshold for the onset of erosion driven by seepage is slope dependent. The threshold disappears at a critical slope $s_{\mathrm{c}}$ determined by the critical Shields number for overland flow and the density contrast between the granular material and water. In most cases this critical slope is significantly smaller than the maximum angle of stability. We find, therefore, that slopes above this critical slope are unstable to any amount of seepage. As a consequence, slopes that sustain seepage must be inclined at an angle smaller than the critical angle for seepage erosion. This behavior contrasts strongly with the threshold phenomena in erosion by overland flow, and therefore provides a mechanistic foundation for distinguishing the two types of erosion.

\section{Acknowledgements}

This work was supported by a DOE Grants DE-FG0299ER15004 and DE-FG02-02ER15367.

We thank N. Schörghofer, W. Dietrich, and K. Whipple for helpful discussions.

\section{References}

Aharonson, O., M. T. Zuber, D. H. Rothman, N. Schorghofer, and K. X. Whipple, Drainage basins and channel incision on Mars, Proceedings of the National Academy of Sciences USA, 99, 1780-1783, 2002.

Baker, V. R., The channels of Mars, University of Texas Press, Austin, 1982.

Baker, V. R., Spring sapping and valley network development, in Groundwater Geomorphology: The role of subsurface water in earth-surface processes and landforms, edited by C. Higgins and D. Coates, chap. 11, Geological Society of America, Boulder, Colorado, 1990.

Buffington, J. M., and D. R. Montgomery, A systematic analysis of eight decades of incipient motion studies, with special reference to gravel-bedded rivers, Water Resources Research, 33(8), 1993-2029, 1997.

Dietrich, W. E., C. J. Wilson, D. R. Montgomery, J. McKean, and R. Bauer, Erosion thresholds and land surface morphology, Geology, 20, 675-679, 1992.

Dunne, T., Formation and controls of channel networks, Progress in Physical Geography, 4, 211-239, 1980.

Dunne, T., Hydrology, mechanics, and geomorphic implications of erosion by subsurface flow, in Special Paper 252, pp. 1-28, Geological Society of America, Boulder, CO, 1990.

Dunne, T., K. X. Whipple, and B. F. Aubry, Microtopography and hillslopes and initiation of channels by Horton overland flow, in Natural and Anthropogenic Influences in Fluvial Geomorphology, pp. 27-44, American Geophysical Union, 1995.

Duran, J., Sands, powders, and grains: an introduction to the physics of granular materials, Springer-Verlag, New York, 1999.

Gomez, B., and V. T. Mullen, An experimental study of sapped drainage network development, Earth Surface Processes and Landforms, 17, 465-476, 1992.

Higgins, C. G., Drainage systems developed by sapping on Earth and Mars, Geology, 10, 147-152, 1982.

Horton, R. E., Erosional development of streams and their drainage basins; hydrophysical approach to quatitative morphology., Geological Society of America Bulletin, 56, 275-370, 1945.
Howard, A. D., Groundwater sapping experiments and modelling, in Sapping Features of the Colorado Plateau, a Comparative Planetary Geology Field Guide, edited by A. D. Howard, R. C. Kochel, and H. E. Holt, pp. 71-83, NASA Scientific and Technical Information Division, Washington, D.C., 1988.

Howard, A. D., A detachment-limited model of drainage basin evolution, Water Resources Research, 30, 2261-2285, 1994.

Howard, A. D., and C. F. McLane, Erosion of cohesionless sediment by groundwater seepage, Water Resources Research, 24, 1659-1674, 1988.

Iverson, R. M., and J. J. Major, Groundwater seepage vectors and the potential for hillslope failure and debris flow mobilization, Water Res. Research, 22(11), 1543-8, 1986.

Izumi, N., and G. Parker, Inception of channelization and drainage basin formation: upstream-driven theory, J. Fluid Mech., 283, 341-363, 1995.

Izumi, N., and G. Parker, Linear stability analysis of channel inception: downstream-driven theory, J. Fluid Mech., 419, 20338,2000 .

Kochel, R. C., and J. F. Piper, Morphology of large valleys on Hawaii - evidence for groundwater sapping and comparisons with Martian valleys, J. Geophys. Res., 91(B13), E175-92, 1986.

Kochel, R. C., A. D. Howard, and C. McLane, Channel networks developed by groundwater sapping in fine-grained sediments: analogs to some Martian valleys, in Models in Geomorphology, edited by M. J. Woldenberg, pp. 313-341, Allen and Unwin, Boston, 1985.

Kochel, R. C., D. W. Simmons, and J. F. Piper, Groundwater sapping experiments in weakly consolidated layered sediments: a qualitative summary, in Sapping Features of the Colorado Plateau, a Comparative Planetary Geology Field Guide, edited by A. D. Howard, R. C. Kochel, and H. E. Holt, pp. 84-93, NASA Scientific and Technical Information Division, Washington, D.C., 1988.

Laity, J. E., and M. C. Malin, Sapping processes and the development of theater-headed valley networks on the Colorado plateau, Geol. Soc. Am. Bull., 96(2), 203-17, 1985.

Landau, L. D., and E. M. Lifshitz, Fluid mechanics, Pergamon Press, Oxford, England; New York, 1987.

Loewenherz, D. S., Stability and the initiation of channelized surface drainage: a reassessment of the short wavelength limit, Journal of Geophysical Research, 96, 8453-8464, 1991.

Martin, C. S., and M. M. Aral, Seepage force on interfacial bed particles, J. Hydrol. Div., HY 7, 1081-1100, 1971.

Montgomery, D. R., and W. E. Dietrich, Channel initiation and the problem of landscape scale, Science, 255, 826-830, 1992.

Montgomery, D. R., and W. E. Dietrich, A physically based model for the topographic control on shallow landsliding, Water Resources Research, 30, 1153-1171, 1994.

Owoputi, L. O., and W. J. Stolte, The role of seepage in erodability, Hydrol. Process., 15, 13-22, 2001.

Schörghofer, N., B. Jensen, A. Kudrolli, and D. H. Rothman, Spontaneous channelization in permeable ground: theory, experiment, and observation, J. Fluid Mech., 503, 357-74, 2004.

Schumm, S. A., M. P. Mosley, and W. E. Weaver, Experimental Fluvial Geomorphology, Wiley, New York, 1987.

Shields, A., Anwendung der Ähnlichkeitsmechanik und der Turbulenzforschung auf die Geschiebebewegung, Heft 26, Mitteilung der Preussischen Versuchsanstalt für Wasserbau und Schiffbau, Berlin, Germany, (In German), 1936.

Smith, T. R., and F. P. Bretherton, Stability and the conservation of mass in drainage basin evolution, Water Resources Research, 3, 1506-28, 1972.

Taylor, D. W., Fundamental of soil mechanics, chap. 16, fifteenth ed., John Wiley \& Sons, Inc., New York, 1965.

Yalin, M. S., Mechanics of sediment transport, 2nd ed., Pergamon Press, Oxford, New York, 1977.

Alexander E. Lobkovsky, Department of Earth, Atmospheric and Planetary Sciences,

MIT, Cambridge, MA 02139, USA, <leapfrog@mit.edu>. 